\title{
Drowning and sudden cardiac death
}

\section{Kenny, R Martin}

Drowning is one of the leading causes of accidental death in children. Some apparent drownings may be related to sudden cardiac death, in particular to unidentified channelopathies, which are known to precipitate fatal arrhythmias during swimming-related events. In this article, the authors examine the likely incidence of such events, the impact of these events on the community, the cardiac defects involved and whether realistic and reliable measures are available to identify those at risk.

In developed countries, drowning is the third leading cause of accidental childhood death with a mortality in the UK of $0.7 / 100000$ children $<15$ years. ${ }^{1}$ The death rate is higher in countries such as the USA and Australia where warmer climates lend themselves to more water exposure and higher numbers of domestic pools. ${ }^{12}$ Data from national agencies in the UK and Australia indicate that $40 \%$ of these drownings occur in swimming pools ${ }^{3}$ while reports from the USA demonstrate that $19 \%$ of drowning deaths in children occur in public pools with certified lifeguards present. ${ }^{5}$ There is less information concerning morbidity, but it is estimated that for each drowning death, there are up to four non-fatal drowning events requiring hospitalisation. ${ }^{1}$ In almost all cases, these events are considered accidental; thus, extensive efforts have been made to reduce the potential for unsupervised and unsafe exposure of young children to water. Drowning rates have consequently declined over recent years predominantly due to these preventive efforts. ${ }^{6}$ However, despite these efforts, one report has recently highlighted that up to $50 \%$ of drownings occur in 5-19year-olds who were are least moderate swimmers. ${ }^{7}$

Bristol Congenital Heart Centre, Bristol Royal Hospital for Children, Bristol, UK

Correspondence to Dr Robin Martin, Consultant Paediatric Cardiologist, Department of Congenital Heart Disease, Bristol Royal Hospital for Children, Upper Maudlin Street, Bristol BS2 8BJ, UK; rob.martin@uhbristol.nhs.uk
Numerous media reports have highlighted sudden demise in young children and adolescents during routine-monitored swimming lessons reflecting high profile cases of sudden deaths seen in athletes in various other sports. These cases have led to charges of negligence brought against lifeguards in swimming pools in the UK for not identifying those in trouble. Cases have been reported indicating that this may not be justified. This includes the case of a reasonable swimmer in supervised swimming lessons in the shallow end of a swimming pool becoming motionless following diving relays and, despite full cardiopulmonary resuscitation for documented pulseless ventricular tachycardia, could not be resuscitated (personal experience). Such cases demonstrate that some apparent drownings may be related to a sudden cardiac event and as such are to some extent immune to the safety measures that have been implemented to date. Highlighting awareness of these conditions may be the first step in limiting the misdiagnosis of some cases of accidental drowning and the consequences this may have on those involved, including family, due to the inheritable nature of these conditions.

\section{SUDDEN CARDIAC DEATH}

Sudden cardiac death occurs in approximately 1 per 100000 person years in young adults. 89 Almost half of these occur in school-aged children ${ }^{10}$ with an estimated overall risk in apparently normal children and adolescents of 1 in $250000 .{ }^{11}$ Thus, the overall risk is low but the impact is significant. The majority of cases are secondary to either hypertrophic or right ventricular cardiomyopathy with coronary artery abnormalities also prevalent, ${ }^{12} 13$ and reports have demonstrated these cardiac abnormalities on autopsy following sudden swimming-related deaths. ${ }^{14}$ However, the majority of autopsies in swimmingrelated sudden deaths are normal suggesting causation at molecular level, in particular ion channel defects such as type 1 long-QT syndrome (LOT1) and catecholaminergic polymorphic ventricular tachycardia (CPVT). ${ }^{13}$ In a recent comprehensive analysis of 1866 sudden deaths in the USA over a 27-year period, 1049 of these deaths were thought to be due to cardiovascular causes. ${ }^{15}$ Of these, 16 occurred while the patient was submerged in water representing just over $1.5 \%$ of all cases. In a similar review over a 13-year period in the UK, 3 of 118 deaths $(2.5 \%)$ occurred during swimming, all of whom had a structurally normal heart. ${ }^{13}$ In these large cohort studies, genetic channelopathies, which include those conditions known to be associated with swimming-related sudden death account for less than $5 \%$ of sudden cardiac death ${ }^{13} 15$ although both studies cited $20-30 \%$ of cases thought to be secondary to a cardiac cause with structurally normal hearts some of whom may also have an ion channel defect.

Approaching this issue from another angle, the prevalence of LQTS is approximately 1 in 5000, with LQT1 accounting for approximately $70 \%$ of genotyped

\section{Box 1 Summary of proposed implementations to improve detection and} appropriate management of apparent drownings secondary to cardiac channelopathies

- Improving awareness in the coronial service of the possibility of a cardiac cause for poorly explained drownings.

- Education of lifeguards and provision of automated defibrillators in swimming pools.

- Molecular autopsy for non-survivors to look for potential channelopathies.

- Screening for survivors and family members of non-survivors to identify those with a channelopathy.

- Proper counselling for those identified to have a channelopathy on family screening. 
LQTS cases. Reports have suggested that swimming may trigger events in between $10 \%$ and $15 \%$ of children and young adults with symptomatic LQTS. ${ }^{16}{ }^{17}$ CPVT although less prevalent than LQTS may affect up to 1 in 10 000, and a recent study suggested that up to $20 \%$ of swimming-related cases with an ion channel defect may be related to CPVT. ${ }^{16}$ Taking these figures into consideration, it is possible that 1 in 100000 individuals may be at risk of a sudden cardiac-related swimming event.

\section{Channelopathies associated with swimming-related events}

LQT1 is caused by a mutation in the KQT-like voltage-gated potassium channel-1 gene (KCNO1; fig 1). This leads to a decrease in the slowly acting repolarising potassium currents and an overall delay in repolarisation with prolongation of the QT interval on surface ECG recordings. Specific triggers for arrhythmia have been associated with particular genotypes (noise and LQT2, sleep and LQT3) with some reports suggesting that swimming-triggered cardiac events are almost pathogonomonic for LQT1. ${ }^{18}$ It is likely that the combination of activities involved in swimming including exertion, cold-water exposure and facial immersion lead to a dominance of sympathetic drive over normal parasympathetic dominance seen during resting state. When heart rate increases quickly with increases in sympathetic activity, reduced rectifying potassium channel activity prevents the necessary QT shortening and early ventricular repolarisation. Premature ventricular contractions, an increased prevalence of which have been identified with swimming, ${ }^{19}$ may occur during the vulnerable part of repolarisation initiating a ventricular arrhythmia. It has also been documented that facial immersion in cold water lengthens the OT interval even in normal subjects ${ }^{20}$ again increasing the length of repolarisation and the susceptibility to early ventricular contractions. This sequence of events has been documented and reported in a child with LQTS who had an implantable

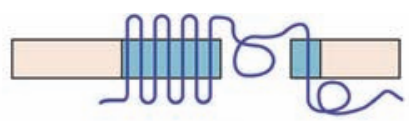

KCNQ1 gene deletion
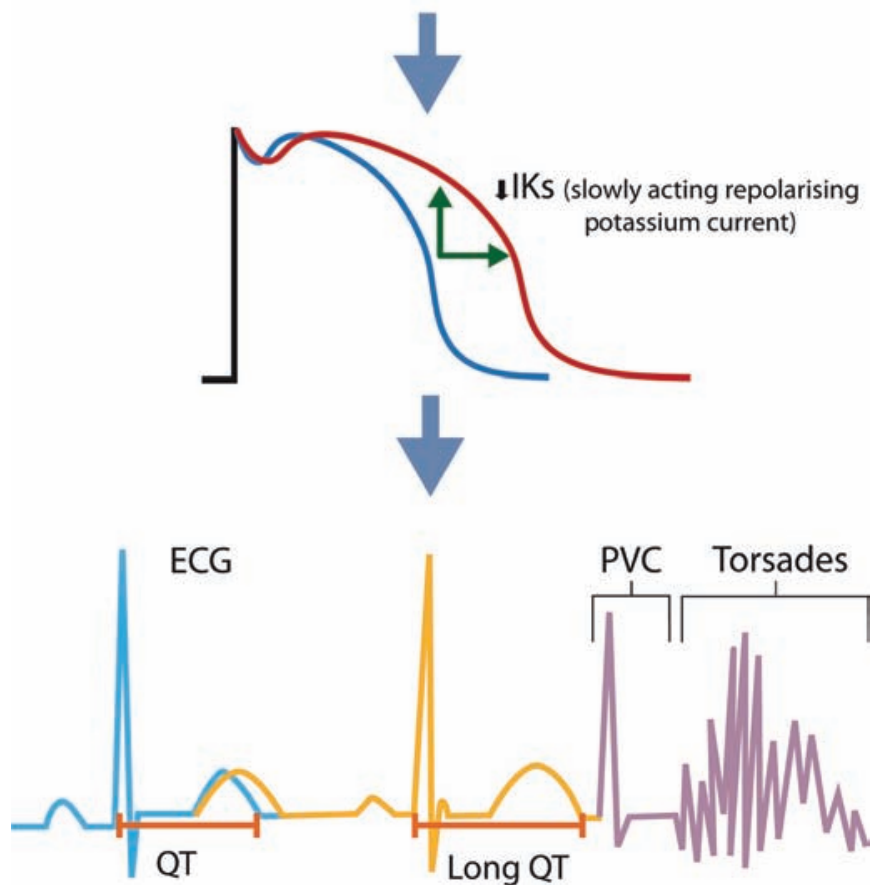

Figure 1 Representation of how long-OT syndrome (LOT1) may lead to swimming-induced arrhythmia. The gene deletion in LOT1 (KCNQ1) leads to a reduction in the repolarising potassium current (IKs) and prolongation of repolarisation. This lengthens the $\mathrm{OT}$ interval (which may be lengthened further by facial immersion in cold water). A premature ventricular contraction (PVC) again which may be initiated by swimming occurring during the vulnerable part of repolarisation leads to establishment of polymorphic ventricular tachycardia (torsades de pointes). cardiac defibrillator in place, while diving head first into the ocean. ${ }^{21}$ The tracings from the implantable defibrillator demonstrated a prolongation of the QT interval following diving into the water followed by a premature ventricular complex which initiated pulseless ventricular tachycardia (fig 2). The reason why some individuals are more at risk than others despite comparable QT intervals may be related to genetic heterogeneity in autonomic responses that occur ${ }^{22}$ such that those with brisk autonomic reflexes may be more at risk of arrhythmia when submerged in cold water.

CPVT is a primary electrical myocardial disease caused by mutations in the cardiac ryanodine receptor channel (RyR2) gene accounting for an autosomal dominant form (CPVT1) in approximately $50 \%$ of cases. The gene defect leads to leakage of calcium from the sarcoplasmic reticulum of the cardiac myocyte across the defective ryanodine receptor leading to calcium overload and delayed afterdepolarisations (fig 3), in contrast somewhat to LQT1 which is associated with early afterdepolarisations. This calcium leakage is accentuated by adrenergic stimulation; hence, the typical exercise induced tachyarrhythmias. The association between CPVT and swimming-related events has been evaluated by Choi et al ${ }^{16}$ who found that 9 out of 10 patients with low suspicion for LQTS following swimming-related events had CPVT1causing RyR2 mutations. From a mechanistic point of view, Choi argues that although swimming may precipitate less acute adrenergic stimulation than other forms of exercise, it is possible that longer R-R intervals seen with swimming may allow for greater diastolic leakage of calcium leading to increased potential for delayed afterdepolarisations although this is speculative. Irrespective of mechanism, the risk of a swimmingrelated event in patients with CPVT is significant, and subsequent reports have confirmed RyR2 mutations in children undergoing molecular autopsy following unexplained drowning. ${ }^{23}$

Although LQT1 and CPVT are the ion channelopathies most significantly associated with swimming-related events, others have been described such as LOT ${ }^{16}$ and LQT7. ${ }^{24}$ Indeed, the most comprehensive assessment to date of ion channel gene defects in those with swimming-related events revealed 4 cases of 43 without an identifiable defect despite comprehensive mutational analysis ${ }^{16}$ 


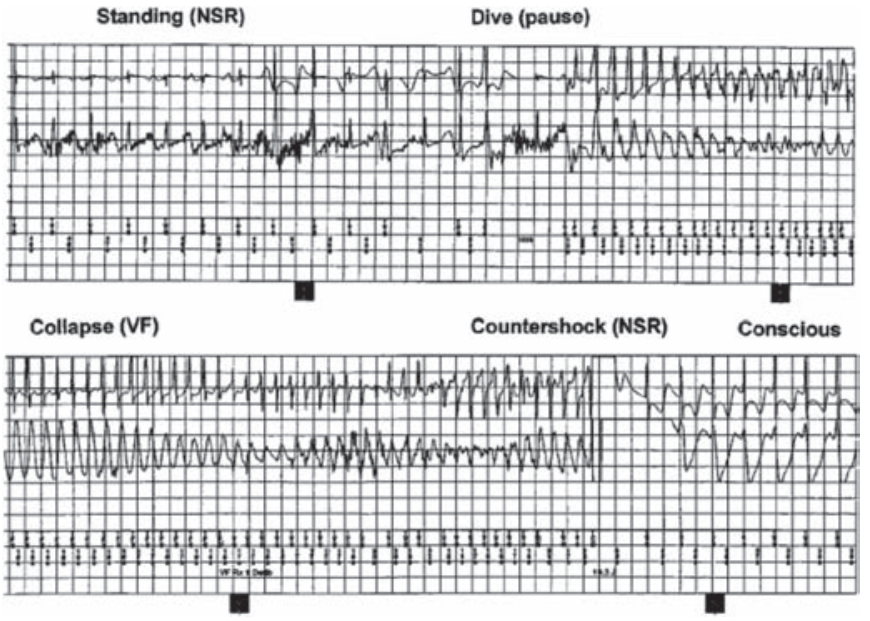

Figure 2 Telemetry recording from an implantable cardiac defibrillator in a 12-year-old girl with long-OT syndrome while diving into cold water. On the top channel, sinus tachycardia is replaced by marked variability in the recording with cold water submersion. The $\mathrm{OT}$ interval is seen to lengthen during this time. The 14th beat is a premature ventricular contraction followed by a pause and then followed by another premature ventricular contraction initiating polymorphic ventricular tachycardia. There is a period of ventricular tachycardia with loss of consciousness, and towards the end of the bottom channel, a DC countershock is delivered restoring sinus rhythm (adapted from Batra and Silka ${ }^{21}$ ).

\section{Ryanodine receptor mutation}

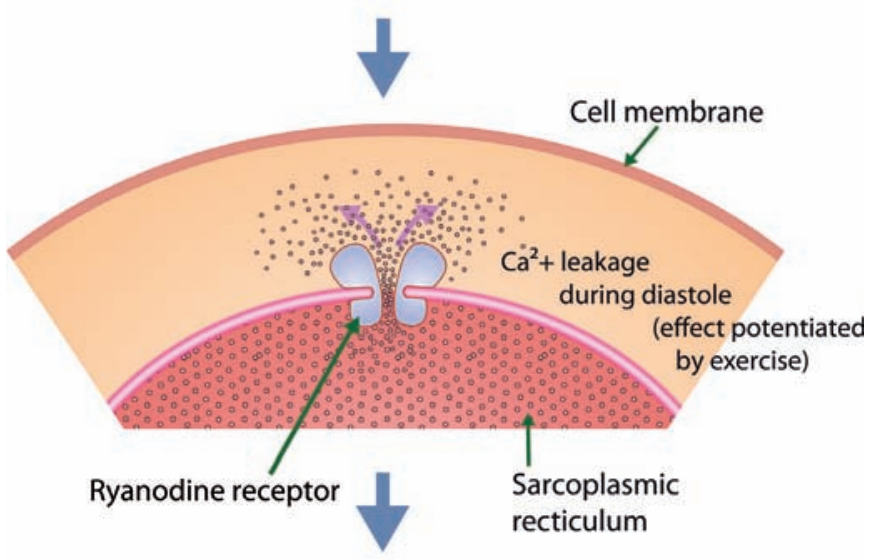

Increased risk for arrhythmia

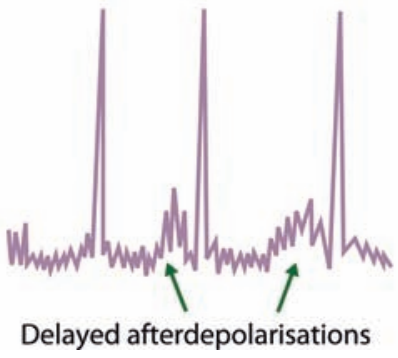

Figure 3 The ryanodine receptor gene mutation (RyR2) in catecholaminergic polymorphic ventricular tachycardia leads to defective closure of the receptor on the surface of the sarcoplasmic reticulum during diastole. This leads to increased calcium $\left(\mathrm{Ca}^{2+}\right)$ leakage from the sarcoplasmic reticulum and increased potential for delayed afterdepolarisations and subsequent ventricular tachycardia. (although this predated newer forms of LOTS) suggesting that there may as yet be unidentified mutations implicated in swimming-related cardiac events.

\section{IDENTIFICATION AND PREVENTION}

Identification of the cause of death in poorly explained drownings may be the first step in a concerted effort at preventing these avoidable events. As the channelopathies involved are inherited, other family members may be at risk; hence, identification of an accurate cause of death may have important implications for the wellbeing of other family members. Molecular autopsy, aimed at detecting disease at genetic level, if comprehensive should detect those ion channelopathies known to be associated with unexplained cardiac deaths including those related to swimming. ${ }^{23} 25$ Unfortunately, widespread understanding of these rare defects may not have reached the coronial service involved. Even should this be the case, in order for the process to be effective, further screening of relatives and subsequent counselling is essential to ensure those at risk are identified, and this requires establishment of a national service to carry out this work. Ideally, a national registry of poorly explained drownings may tap into this service so that these events are thoroughly investigated. This has yet to be achieved in the UK. Greater awareness of these events among lifeguards and provision of external automatic defibrillators to all public swimming areas may reduce subsequent death once an event has occurred as external automatic defibrillators have been demonstrated to be an effective strategy to reduce deaths at high-risk sites. ${ }^{26}$

The ultimate goal, however, which may prove the most challenging, should be to identify those at risk before any event occurring. Preparticipation screening guidelines for children and adolescents involved in sport have been produced by the American Heart Association ${ }^{9}$ and the European Society of Cardiology ${ }^{27}$ differing somewhat in age of screening; requirement for an ECG and whether serial evaluations are necessary. Irrespective of the approach, significant controversy exists on whether such programmes are cost-effective $^{11}$ despite evidence that they may reduce sudden cardiac death in the young. ${ }^{28}$ In relation to identifying those conditions specifically implicated in swimming-related sudden death, the current strategy recommended by the 
European Society of Cardiology might not be reliable and is certainly unlikely to be cost-effective. LOT1 is a disease of childhood, and many events may have occurred before recommended screening age has been reached. Also, up to $30 \%$ of patients with LOTS may not demonstrate a long QT interval on surface ECG ${ }^{29}$ suggesting some cases (although probably lower risk) may be missed. The situation is even worse with CPVT as surface ECG changes are usually not present at rest; thus, it may be almost impossible to identify those at risk without a family history (70\% of cases are de novo mutations) or previous symptoms. Universal screening may also introduce significant false positives with up to $5 \%$ of school children noted to have some abnormality on surface ECG. ${ }^{11}$ This, in itself, could have a negative impact on the child and the families involved. Thus, establishing a sudden cardiac death program with availability of molecular autopsy and subsequent family screening remains the most appropriate initial step. Whether universal screening will be adopted in the UK and whether it will have any impact on these rare but tragic events remains to be seen.

\section{CONCLUSIONS}

Drowning is a common cause of death in childhood; however, some cases may not be accidental and occur secondary to cardiac abnormalities. Misdiagnosing cause of death may have significant implications for family members of the index case and for those involved in guarding our public swimming areas. Greater awareness of these cardiac conditions, particularly the ion channelopathies strongly associated with swimming-related events, may lead to prompt appropriate treatment when an event occurs followed by identification of other family members who may be at risk. In order for this to occur, a national registry of poorly explained drownings is required with links to a national strategic program dealing with sudden cardiac deaths.

Funding This work was funded internally from departmental resources.

\section{Competing interests None.}

Provenance and peer review Commissioned: externally peer reviewed.
Accepted 12 April 2010

Published Online First 28 June 2010

\section{REFERENCES}

1. Kemp A, Sibert JR. Drowning and near drowning in children in the United Kingdom: lessons for prevention. BMJ 1992;304:1143-6.

2. Meyer RJ, Theodorou AA, Berg RA. Childhood drowning. Pediatr Rev 2006;27:163-8; quiz 169.

3. The Royal Society for Prevention of Accidents. http://www.rospa.com/leisuresafety/ statistics/child-accidental-drownings-2005.aspx (accessed Jun 2010)

4. Royal Life Saving Society - Australia. 15th Annual Drowning Report Released by the Royal Life Saving Society - Australia. http://www. royallifesaving.com.au/www/html/2269-2009 national-drowning-report.asp laccessed Jun 2010).

5. Infant Swimming Resource. National Drowning Statistics. http://www.infantswim.com/assets/ docs/ISR Statistics2009.pdf (accessed Jun 2010).

6. Sibert JR, Lyons RA, Smith BA, et al.; Safe Water Information Monitor Collaboration. Preventing deaths by drowning in children in the United Kingdom: have we made progress in 10 years? Population based incidence study. BMJ 2002;324:1070-1.

7. Brenner RA, Taneja GS, Haynie DL, et al. Association between swimming lessons and drowning in childhood: a case-control study. Arch Pediatr Adolesc Med 2009;163:203-10.

8. Corrado D, Basso C, Rizzoli G, et al. Does sports activity enhance the risk of sudden death in adolescents and young adults? J Am Coll Cardiol 2003;42:1959-63.

9. Maron BJ, Thompson PD, Ackerman MJ, et al Recommendations and considerations related to preparticipation screening for cardiovascular abnormalities in competitive athletes: 2007 update: a scientific statement from the American Heart Association Council on Nutrition, Physical Activity, and Metabolism: endorsed by the American College of Cardiology Foundation. Circulation 2007:115:1643-55

10. Maron BJ, Gohman TE, Aeppli D. Prevalence of sudden cardiac death during competitive sports activities in Minnesota high school athletes. J Am Coll Cardiol 1998;32:1881-4.

11. Wren C. Screening for potentially fatal heart disease in children and teenagers. Heart 2009:95:2040-6.

12. Maron BJ, Shirani J, Poliac LC, et al. Sudden death in young competitive athletes. Clinical, demographic, and pathological profiles. JAMA 1996;276:199-204

13. de Noronha SV, Sharma S, Papadakis M, et al Aetiology of sudden cardiac death in athletes in the United Kingdom: a pathological study. Heart 2009:95:1409-14

14. Durakovic Z, Misigoj Durakovic M, Skavic J, et al. Myopericarditis and sudden cardiac death due to physical exercise in male athletes. Coll Antropol 2008;32:399-401

15. Maron BJ, Doerer JJ, Haas TS, et al. Sudden deaths in young competitive athletes: analysis of
1866 deaths in the United States, 1980-2006. Circulation 2009;119:1085-92.

16. Choi G, Kopplin LJ, Tester DJ, et al. Spectrum and frequency of cardiac channel defects in swimmingtriggered arrhythmia syndromes. Circulation 2004;110:2119-24.

17. Moss AJ, Robinson JL, Gessman L, et al. Comparison of clinical and genetic variables of cardiac events associated with loud noise versus swimming among subjects with the long OT syndrome. Am J Cardio/ 1999;84:876-9.

18. Ackerman MJ, Tester DJ, Porter CJ. Swimming, a gene-specific arrhythmogenic trigger for inherited long QT syndrome. Mayo Clin Proc 1999;74:1088-94.

19. Ishikawa H, Matsushima M, Nagashima M, et al. Screening of children with arrhythmias for arrhythmia development during diving and swimming - face immersion as a substitute for diving and exercise stress testing as a substitute for swimming. Jpn Circ J 1992;56:881-90.

20. Yoshinaga M, Kamimura J, Fukushige T, et al. Face immersion in cold water induces prolongation of the $\mathrm{OT}$ interval and T-wave changes in children with nonfamilial long QT syndrome. Am J Cardiol 1999;83:1494-7, A8.

21. Batra AS, Silka MJ. Mechanism of sudden cardiac arrest while swimming in a child with the prolonged OT syndrome. J Pediatr 2002;141:283-4.

22. Schwartz PJ, Vanoli E, Crotti L, et al. Neural control of heart rate is an arrhythmia risk modifier in long QT syndrome. J Am Coll Cardiol 2008; 51:920-9.

23. Tester DJ, Kopplin LJ, Creighton W, et al. Pathogenesis of unexplained drowning: new insights from a molecular autopsy. Mayo Clin Proc 2005;80:596-600.

24. Efremidis M, Pappas LK, Sideris A, et al. Swimming-triggered aborted sudden cardiac death in a patient with Andersen-Tawil syndrome. Int J Cardiol 2006;112:e45-7

25. Ackerman MJ, Tester DJ, Driscoll DJ Molecular autopsy of sudden unexplained death in the young. Am J Forensic Med Pathol 2001;22:105-11

26. Davies CS, Colquhoun MC, Boyle R, et al. A national programme for on-site defibrillation by lay people in selected high risk areas: initial results. Heart 2005;91:1299-302.

27. Corrado D, Pelliccia A, Bjornstad HH, et al. Cardiovascular preparticipation screening of young competitive athletes for prevention of sudden death: proposal for a common European protocol: consensus statement of the Study Group of Sport Cardiology of the Working Group of Cardiac Rehabilitation and Exercise Physiology and the Working Group of Myocardial and Pericardial Diseases of the European Society of Cardiology. Eur Heart J 2005;26:516-24.

28. Corrado D, Basso C, Schiavon M, et al. Preparticipation screening of young competitive athletes for prevention of sudden cardiac death. J Am Coll Cardiol 2008;52:1981-9.

29. Zhang L, Timothy KW, Vincent GM, et al. Spectrum of ST-T-wave patterns and repolarization parameters in congenital long-OT syndrome: ECG findings identify genotypes. Circulation 2000;102:2849-55. 